\title{
The Study of Prevalence of Low Back Pain and Its Correlation with Functional Disability in Patients with Lower Limb Amputation
}

\author{
Arya Shirish Ashtaputre ${ }^{1}$, Ayman Shaad Gondekar², Govinda Varadharajulu ${ }^{3}$ \\ 1,2,3 Department of Physiotherapy, Krishna Institute of Medical Sciences, \\ Deemed to Be University, Karad, Maharashtra, India
}

\section{ABSTRACT}

\section{BACKGROUND}

Low back pain (LBP) is a very common and disabling condition experienced by individuals during their lifetime. However, the causes of low back pain may vary and include musculoskeletal impairments, biomechanical abnormalities, gait deviations, primary medical causes, and deleterious or excessive activity. ${ }^{1}$ The different levels at which amputation is done in the lower limb are hip disarticulation, transfemoral amputation (above knee), knee disarticulation, transtibial amputation (below knee), ankle disarticulation and transmetatarsal amputation. Lower limb amputation (LLA) is known to cause LBP and may result in functional disability in lower limb amputees. Different parameters such as level of amputation, use of prosthesis etc. affect low back pain in amputees. The purpose of this study was to find out the prevalence of low back pain and its correlation with functional disability in patients with lower limb amputation.

\section{METHODS}

This is a community based observational study. 41 participants were included in the study. Data was collected using a questionnaire including the demographic information of each participant, pain assessment and revised Oswestry low back pain disability (RODQ) questionnaire to assess functional disability.

\section{RESULTS}

The study revealed that the prevalence of the reported back pain in the studied sample was $60.9 \%$. In $60 \%$ of the patients, the Oswestry disability index depicted moderate disability.

\section{CONCLUSIONS}

The study concludes that low back pain is a significant problem in lower limb amputees. Several factors such as level of amputation, age, time since amputation put the lower limb amputees at a risk of developing back pain. Back pain intensity is important to assess the back pain related functional disability.

\section{KEY WORDS}

Amputation, Low Back Pain, Disability, Prosthesis
Corresponding Author: Dr. Govinda Varadharajulu, Dean, Department of Physiotherapy, Krishna Institute of Medical Sciences Deemed to be University, Karad, Maharashtra, India.

E-mail: aryaashtaputre@gmail.com

DOI: $10.14260 /$ jemds/2021/457

How to Cite This Article:

Ashtaputre AS, Gondekar AS, Varadharajulu G. The study of prevalence of low back pain and its correlation with functional disability in patients with lower limb amputation. J Evolution Med Dent Sci 2021;10(30):2233-2238, $10.14260 /$ jemds/2021/457 DOI:

Submission 06-05-2021,

Peer Review 04-07-2021, Acceptance 12-07-2021, Published 26-07-2021.

Copyright (C) 2021 Arya Shirish Ashtaputre et al. This is an open access article distributed under Creative Commons Attribution License [Attribution 4.0 International (CC BY 4.0)] 


\section{BACKGROUND}

Amputation of lower limb is more commonly performed than that of upper limb. Injury is the commonest cause of amputation in developing countries. The common causes of injury are peripheral vascular disease in elderly, secondary to injury or its sequel in younger adults, and deficiency of limb since birth in children. The different levels at which amputation is done in the lower limb are hip disarticulation, transfemoral amputation (above knee), knee disarticulation, transtibial amputation (below knee), ankle disarticulation and transmetatarsal amputation. Lower back pain is one of the most frequently reported musculoskeletal problems in persons with disabilities. It is more common in the population of lower limb amputation than in the general population. In lower limb amputees, low back pain is quite bothersome and can also be associated with poor quality of life and depression. There are a number of studies that explore phantom limb pain and residual limb pain in amputees but the investigation of low back pain in lower limb amputees has received minimal attention in literature. Among all the levels, transfemoral amputees are at higher risk for predominantly biomechanical aetiology of low back pain Excessive motion in spine may cause abnormal deformity in musculoskeletal element that may alter normal lumbar spine kinematics. Movement asymmetries are seen in lower limb amputees during walking that could affect time distance parameters, lumbopelvic and lower limb movements. ${ }^{1}$

Chronic low back pain is considered as a secondary disability of amputation. There is evidence in research literature that low back pain in amputees is more bothersome than any other pain. Understanding the potential mechanisms of low back pain will help in the care, management, and longterm rehabilitation process. In amputees, the following biomechanical abnormalities can be considered as the cause of low back pain. (i) asymmetrical movements (ii) abnormal loading patterns and (iii) muscular asymmetries. The absence of joint in amputees leads to altered joint kinematics. Therefore, while doing activities of daily living, there is compensation with the intact musculature which ultimately leads to asymmetrical movements causing secondary muscular disorders. ${ }^{2}$

In the normal population, the causes of low back pain can be repetitive lifting, abnormal posture etc. In amputees, the cause of low back pain could be degeneration, its reason being increased lumbar transverse plane rotation while walking leading to increased shear forces on the disc. Asymmetrical movements lead to prolonged deformation of the passive ligamentous structures in the lumbopelvic region. This causes increased activity of the paraspinal muscles ultimately leading to fatigue and pain. ${ }^{3-6}$ Also, the relationship between low back pain and functional disability has been extensively studied in the general population but there is lack of such studies in amputees. ${ }^{7}$ Causes of low back pain have been studied extensively and include musculoskeletal impairments, biomechanical abnormalities, gait deviation deleterious or excessive activity and inappropriate prosthesis. ${ }^{8}$ Spinal movements, altered gait, asymmetries in daily activities are also contributing factors for low back pain. Several factors such as physical and biomechanical factors (heavy lifting work, awkward postures, repetitive straining), psychosexual factors (anxiety, depression, job dissatisfaction) and personal factors (age, sex, mass, level of amputation, type of prosthesis, amount of daily exercise, smoking) have been heavily debated in contributing to low back pain among all amputee populations. ${ }^{9}$

Traumatic amputation is common in younger age group and these patients are more active prosthetic users. In transtibial amputees, the success rate is higher than that of transfemoral amputees. Traumatic amputees tend to be younger and fitter than vascular disease and diabetic amputees. Thus, they are more capable of recovering from their injury to a state where they are independently ambulating and achieving high levels of functioning in daily activities. After intensive physiotherapy, the success rate of using the prosthesis is high. There is a significant association of physical activity (PA) and low back pain. After amputation, patients find difficulty in performing activities of daily living as the patients have to adapt to their artificial limb which takes longer duration due to somatosensory loss.

Lower limb amputees with chronic back pain report significantly more disability than those without back pain. Prosthesis is best known management for correcting posture, strengthening of back muscles, and reducing pain disability. Most commonly used scale of disability for persons with chronic low back pain is Roland-Morris-disability and revised Oswestry low back pain disability (RODQ) questionnaire. The results of this study will add to the understanding of low back pain and its association with functional disability in lower limb amputees.

\section{Objectives}

1. To determine the prevalence of low back pain in amputees.

2. To correlate the self-reported functional disability levels with low back pain.

3. To explore the relationship between LBP and restricted activities of daily living (ADL).

4. To characterise the relationship between LBP and amputation related parameters.

\section{METHODS}

The study design was community based observational study conducted in Karad. The ethical clearance to conduct the study was provided by the Institutional Ethical Committee of Krishna Institute of Medical Sciences, Deemed to be University, Karad (protocol number 0117). It was done by taking interviews of patients based on questionnaires. We carried out the interview according to the questionnaire either in the home of the patient or at the hospital as preferred by the patient. The questions were grouped into: Demographic data and assessment data including visual analogue scale and the questionnaire (Revised Oswestry low back pain disability questionnaire). The data was collected within duration of 6 months from November 2019 to April 2020. Participants were selected from the Orthotics and Prosthetic Department of Krishna Institute of Medical Sciences, Deemed to be University, Karad and were evaluated according to the inclusion and exclusion criteria. Amputees with low back pain before their amputation, those who had 
injured their back in the past and those with previous back surgery were excluded. The inclusion criteria were persons with unilateral lower limb amputation, post amputation 6 months and all levels of amputation, irrespective of the age of the amputees of lower limb. 41 amputees participated and the following assessment was taken

Formula $n=4 p q / I^{2}$

[where, $\mathrm{p}=64, \mathrm{q}=36$ and $\mathrm{l}=10]^{8}$

The questionnaire consisted of 3 domains:

1. Demographics and history taking.

2. Low back pain assessment (Visual Analogue Scale) ${ }^{9}$

3. Physical activity related questionnaire (revised Oswestry low back pain disability questionnaire) ${ }^{10}$

After taking informed consent from the participants their demographic data was noted. The demographic data included

Name:

Age:

Gender:

Occupation:

Address:

Height:

Weight:

BMI:

Date of amputation:

Date of assessment:

Assessment data

1. Do you suffer from low back pain?

$\square$ yes $\square$ no

2. Do you use prosthesis / artificial limb?

$\square$ yes $\square$ no

3. If yes, then since when?

\section{Measures}

\section{Visual Analogue Scale}

Intensity of low back pain at rest and on activity was measured using the visual analogue scale. It is a valid, simple and reliable tool for measuring pain intensity. Visual analogue scale consists of $10 \mathrm{~cm}$ line, with the left extremity indicating 'no pain' and the right extremity indicating 'severe pain'. Patients were asked to rate their current level of low back pain on this scale.

\section{Revised Oswestry Low Back Pain Disability Questionnaire (RODQ)}

All the participants with LBP filled the revised Oswestry low back pain disability questionnaire. It is a clinical assessment tool that provides an estimate of disability expressed as a percentage score. The English and Marathi version of the questionnaire was used according to the convenience of the patients. The questionnaire consists of 10 sections (pain intensity, personal care, lifting, walking, sitting, standing, sleeping, sex life, social life and travelling). Each section has 6 questions with score ranging from $0-5$. The scores are totalled, and disability percentage is calculated. Higher the score greater is the disability. The scores are ranging from 0 $100 \%$ were graded as following:

- $0 \%$ to $20 \%$ - minimally disabled
- $21 \%$ to $40 \%$ - medium disabled

- $41 \%$ to $60 \%$ - seriously disabled

- $61 \%$ to $80 \%$ - badly disabled

- $81 \%$ to $100 \%$ - extremely disabled (totally immobile)

\section{Statistical Analysis}

Statistical analysis was done under window using statistical package for social sciences (SPSS) program, V10. Descriptive statistics are presented as means with standard deviation (SD) Comparison was done using 'paired t' test. Correlation study between low back pain and functional disability was done by using 'Pearson correlation' test.

\section{RESULTS}

In the studied sample, the age of the lower limb amputees were ranging from 23 to 65 years. $26.8 \%$ of the studied samples were female and $73.1 \%$ were male. $34 \%$ of the amputees were transfemoral, $51 \%$ were transtibial, $7.3 \%$ were through knee and $7.3 \%$ were ankle disarticulation. 87.8 $\%$ of the amputees were using prosthesis and $12.1 \%$ were not using prosthesis. The prevalence of the reported back pain in the studied sample was $60.9 \%$. The mean of back pain intensity on visual analogue scale was $4.3 \pm 1.4$ at rest and 6.1 \pm 1.3 on activity. The transfemoral amputees are at a higher risk of development of back pain. The use of prosthesis may also have an effect on low back pain. Among the patients, who are using prosthesis (87.81\%), $38.98 \%$ patients suffer from low back pain. and remaining $48.73 \%$ patients do not have LBP. All the patients (12.19\%) who do not use prosthesis suffer from low back pain.

In $60 \%$ of the patients, the Oswestry disability index depicted moderate disability. Majority of the patients with mild pain had moderate disability and majority of those with moderate pain had severe disability. None of the patients documented minimum disability or were crippled.

\begin{tabular}{|ccc|}
\hline Level of Amputation & VAS (at Rest) & VAS (on Activity) \\
Above knee & $4.3(0.8)$ & $6.5(0.6)$ \\
Below knee & $4.3(1.6)$ & $6.0(1.5)$ \\
Through knee & $6.1(0.0)$ & $7.6(0.0)$ \\
\hline Table 1. Comparison between Intensity of Low Back Pain in Rest and \\
on Activity with Levels of Amputation Values are Expresses as Mean \\
\\
(SD)
\end{tabular}

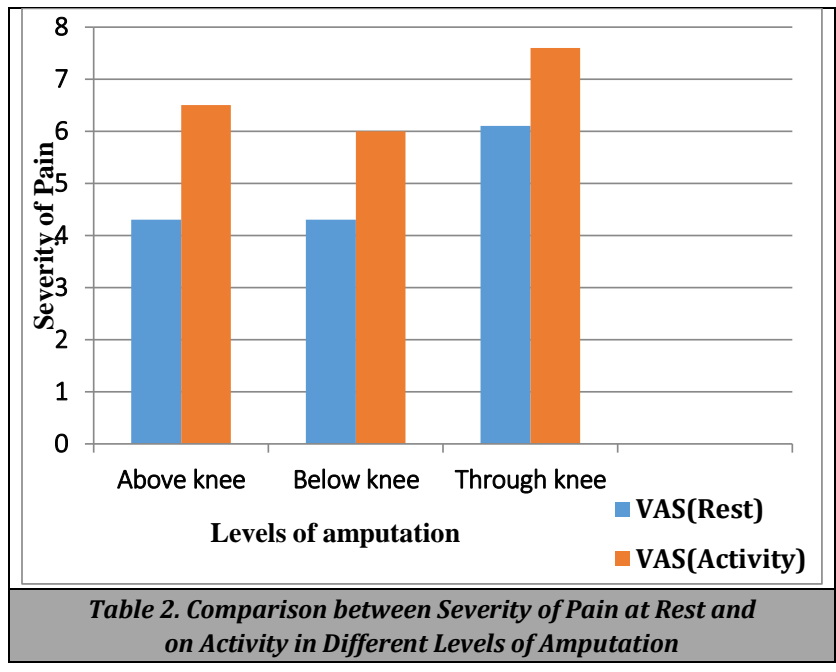


The above graph represents intensity of low back pain at rest and on activity in different levels of amputation (above knee, below knee and through knee). The visual analogue scale on activity is significantly higher than visual analogue scale at rest in all levels of amputation. The intensity of pain is maximum in patients with through knee amputation and comparatively minimum in patients with below knee amputation.

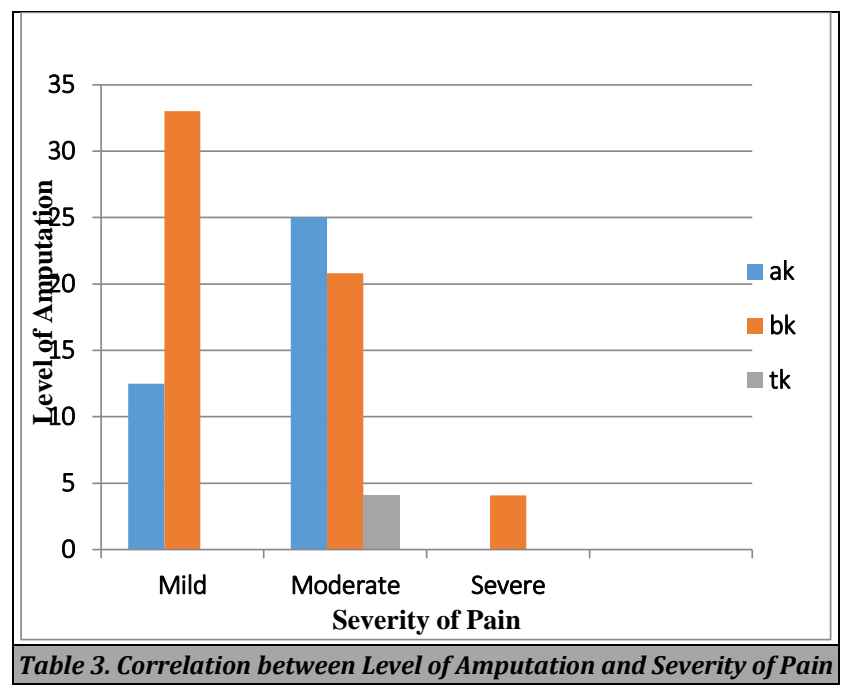

The above graph represents percentage number of amputees (above knee, below knee, through knee) who are suffering from mild, moderate, and severe pain respectively. $12.5 \%$ of patients with above knee amputation have mild pain and $25 \%$ of them have moderate pain. $33 \%$ of patients with below knee amputation have mild pain, $20.8 \%$ have moderate pain and $4.1 \%$ have severe pain. $4.1 \%$ of patients with through knee amputation have moderate pain.

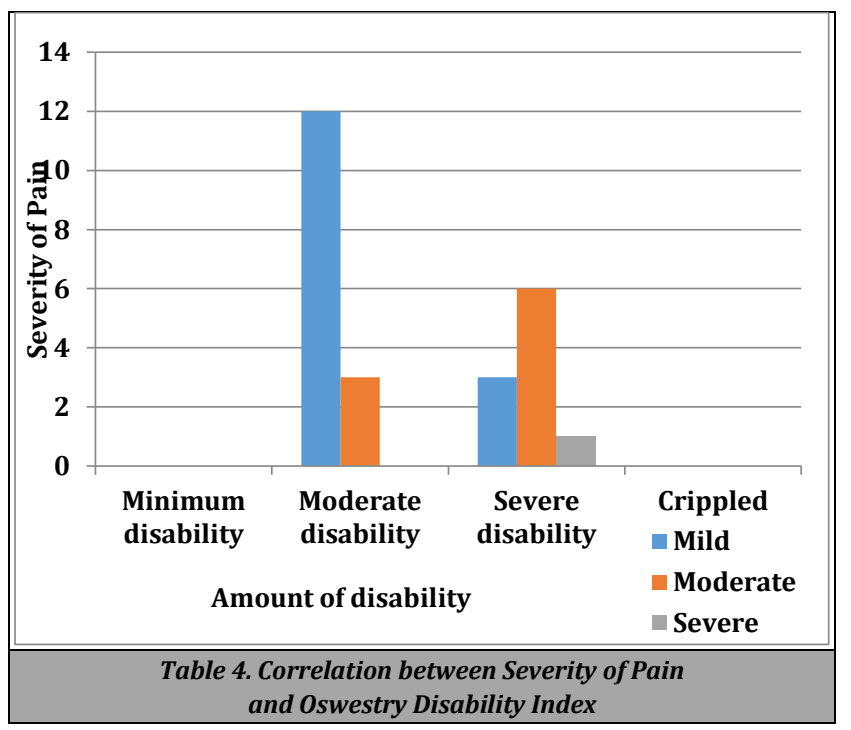

The above graph represents the correlation between the severity of pain and amount of disability (according to revised Oswestry low back pain disability questionnaire). 12 $\%$ of patients with mild pain documented moderate disability and $3 \%$ of them documented severe disability. $3 \%$ of patients with moderate pain documented moderate disability and $6 \%$ of them documented severe disability. $1 \%$ of patients with severe pain documented severe disability.
There were no patients with minimum disability and none of them were crippled.

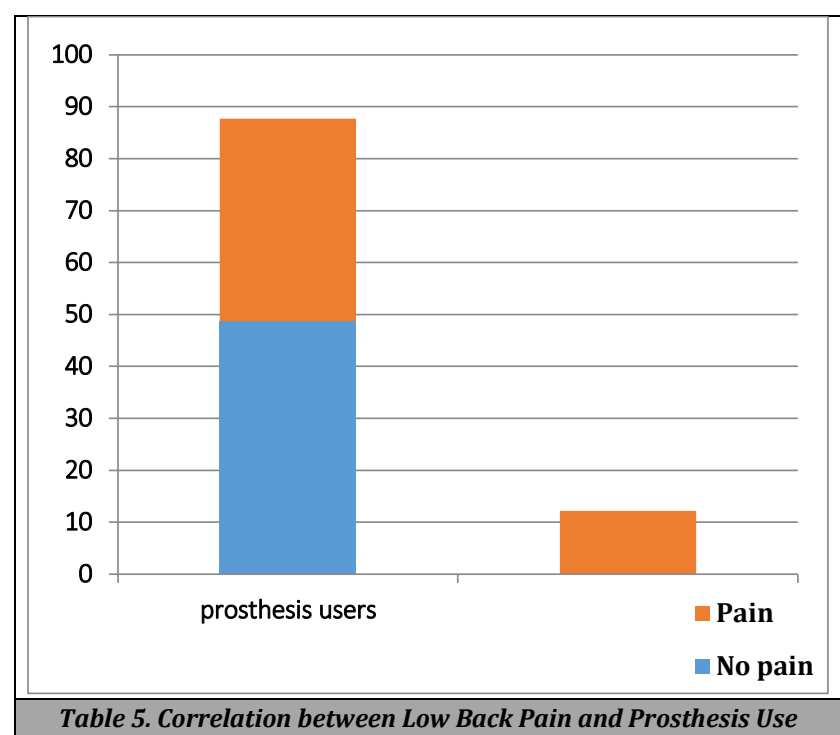

The above graph represents correlation between low back pain and use of prosthesis in patients with lower limb amputation. It shows that among the patients, who are using prosthesis (87.81\%), $38.98 \%$ patients suffer from low back pain and remaining $48.73 \%$ patients do not have low back pain. All the patients (12.19\%) who do not use prosthesis suffer from low back pain. There is decreased risk of developing low back pain with increasing duration of use of prosthesis.

\section{DISCUSSION}

According to the results of the study, it was confirmed that post amputation back pain is a prevalent problem among lower limb amputees. Among the studied sample, $60.9 \%$ of the patients reported presence of back pain post amputation. This prevalence is higher than that of the general population. In a previous study, Smith et al. (1999) have found that low back pain is more bothersome than phantom limb pain or residual limb pain. Kulkarni et al. (2005) concluded that low back pain in lower limb amputees was a significant problem equal to that of pain in the phantom limb and a biomechanical rather than a degenerative aetiology is suggested. ${ }^{8}$

In the present study, the prevalence of back pain was found to be significant in lower limb amputees. Majority of the patients reported their back-pain intensity in the moderate range. This finding is similar to that of Amal Bakry Ahmad Abdul Sattar (2007) who stated that half of the studied lower limb amputees reported average of their backpain intensities in the moderate range. The present study found that transfemoral amputation was associated with a higher risk of development of back pain than transtibial amputation. But there was no significant variation in the intensities of back pain in above knee and below knee amputees. Smith et al. (1999) has also found that pain is significantly more frequent, intense, and bothersome in persons with above knee amputations compared with below knee amputations. Presence of other problems such as 
phantom limb pain and residual limb pain may increase the risk of development of back pain. ${ }^{8}$

The present study also found a significant positive correlation between the intensity of back pain in lower limb amputees and back pain related functional disability. This result agrees with that of Marshall et al. (2002) who found that lower limb amputees with chronic back pain report significantly more disability than lower limb amputees without back pain. ${ }^{8}$

Functional disability was measured using the revised Oswestry low back pain disability (RODQ) questionnaire. However, the use of questionnaire may have caused some disadvantage and decreased the participation of the patients as those with little or no pain may have thought that the questions do not concern them. The understanding of the wordings of the items in the questionnaire may also have caused decreased responses or error in the responses to the questionnaire. Thorough explanation of the need for and importance of study may have increased the participation. ${ }^{11}$

Amputees also go through other related problems such as stump pain or phantom limb pain. There is a possibility of these problems being more prominent than perceived back pain at the time of filling the questionnaire. ${ }^{11}$ Similar to the study done by Hammarlund et al. (2010) in the present study the prevalence of back pain was rated retrospectively by the participants, and as many years had elapsed since amputation this may have caused some unreliability in the data. ${ }^{11}$ In the article given by Hemakumar Devan the physical activity and intensity of low back pain is only measured in the patients who have undergone trans femoral amputation. But in our study, all the levels of amputation were included.7 In the previous studies, it is stated that as the age of lower limb amputees increased, the odds of developing low back pain increased, but in the present study no such conclusion was made.

Use of prosthesis also has an effect on low back pain. In our study, all the patients who were not using prosthesis suffered from low back pain. But in those using prosthesis the prevalence of low back pain was less. The type of prosthesis may also have an impact on low back pain but the influence of type of prosthesis worn was not investigated in our study. A previous study has found back pain to be more prevalent in lower socioeconomic groups. Back pain also differs according to the occupational groups of the subjects. ${ }^{12}$ In our study, the subjects were not categorised according to their socioeconomic or occupational backgrounds. Therefore, such conclusions could not be made.

Increased body mass index (BMI) has also been linked with low back pain. ${ }^{12}$ But in our study, no such conclusions could be made. Patients also reported an uneven manner while doing their daily activities due to artificial limb, architectural barriers, irregular surface, obstacles in a crowded situation and other injuries during accident also contributed to uneven posture and compensatory movements of lower limb and back. Results given by Friel et al. show an association between low back pain and functional scores as measured by the revised Oswestry low back pain disability questionnaire. Our study also revealed a positive correlation between severity of back pain and self-reported functional disability. There may also be some other limitations of the study. The sample size is small, and the subgroups are of unequal size, therefore the results may not be generalizable. Further studies are needed to evaluate the relationships between pain, health related quality of life, psychological aspects, and functional capacity. Further studies can also be done to investigate the possible mechanisms of low back pain in lower limb amputees. Gait impairments can also be a contributing factor to low back pain in lower limb amputees, majority of the subjects reported phantom pain residual limb pain, skin problems in the residual limb as a contributing factor in gait impairment. ${ }^{2}$ Therefore, studies exploring abnormalities in gait and its possible effects on functional disability in lower limb amputees are suggested.

\section{CONCLUSIONS}

The study concludes that low back pain is a significant problem in lower limb amputees. Several factors such as level of amputation, age, time since amputation put the lower limb amputees at a risk of developing back pain. Back pain intensity is important to assess the back pain related functional disability. Back pain influences functional abilities in greater extent in lower limb amputees. Also, use of prosthesis may also reduce low back pain and functional disabilities in lower limb amputees. Poor clinical outcome is seen in patients with acute low back pain post amputation that addressed and identified the casual belief of patients toward their back pain and its management.

\section{Limitations}

The primary limitation of the study was the number of participants recruited. The sample size was small and the subgroups were of unequal size. Therefore, the results could not be generalized. In further studies, the relationship between pain, quality of life, psychological aspects and functional capacity can be evaluated.

Data sharing statement provided by the authors is available with the full text of this article at jemds.com.

Financial or other competing interests: None.

Disclosure forms provided by the authors are available with the full text of this article at jemds.com.

We would like to thank Dr. Kashinath Sahoo, Head of Department of Prosthetics and Orthotics, Krishna Institute of Medical Sciences Karad deemed to be university for his valuable assistance and support. We would also like to thank all the participants for sharing their experience.

\section{REFERENCES}

[1] Abdul-Sattar ABA. Amputation-related back pain: prevalence, associated risk factors and correlation with functional disability. Egypt Rheumatol Rehab 2007;34(2):183-96.

[2] Hemakumar D, Tumilty S, Smith C. Physical activity and lower-back pain in persons with traumatic transfemoral amputation: a national cross-sectional survey. J Rehab Res Dev 2012;49(10):1457-66.

[3] Hammarlund CS, Carlstorm M, Melchior R, et al. Prevalence of back pain, its effect on functional ability 
and health-related quality of life in lower limb amputees secondary to trauma or tumour: a comparison across three levels of amputation. Prosthet Orthot Int 2011;35(1):97-105.

[4] Kulkarni J, Gaine WJ, Buckley JG, et al. Chronic low back pain in traumatic lower limb amputees. Clin Rehab 2005;19(1):81-6.

[5] Sivapuratharasu B, Bull AMJ, McGregor AH. Understanding low back pain in traumatic lower limb amputees: a systematic review. Arch Rehabil Res Clin Transl 2019;1(1-2):100007.

[6] Friel K, Domholdt E, Smith DG. Physical and functional measures related to low back pain in individuals with lower-limb amputation: an exploratory pilot study. J Rehabil Res Develop 42.2 2005;42(2):155-66.

[7] Kušljugić A, Kapidzic-Durakovic S, Kudumovic Z, et al. Chronic low back pain in individuals with lower-limb amputation. Bosn J Basic Med Sci 2006;6(2):67-70.
[8] De Fretes A, Boonstra AM, Vos LD. Functional outcome of rehabilitated bilateral lower limb amputees. Prosthet Orthot Int 1994;18(1):18-24.

[9] Hendershot BD, Bazrgari B, Nussbaum MA. Persons with unilateral lower-limb amputation have altered andasymmetric trunk mechanical and neuromuscular behaviors estimated using multidirectional trunk perturbations. J Biomech 2013;46(11):1907-12.

[10] Devan H, Hendrick P, Ribeiro DC, et al. Asymmetrical movements of the lumbopelvic region: is this a potential mechanism for low back pain in people with lower limb amputation? Med Hypotheses 2014;82(1):77-85.

[11] Page SJ, Shawaryn MA, Cernich AN, et al. Scaling of the revised oswestry low back pain questionnaire. Arch Phys Med Rehabil 2002;83(11):1579-84.

[12] Boonstra AM, Preuper HRS, Reneman MF, et al. Reliability and validity of the visual analogue scale for disability in patients with chronic musculoskeletal pain. Int J Rehabil Res 2008;31(2):165-9. 